\title{
GENEALOGÍA DE LA TORTURA EN COLOMBIA: UNA MIRADA DESDE LOS DERECHOS HUMANOS
}

Paola Alexandra Sierra-Zamora

Lina Vanessa Jiménez-Barrera

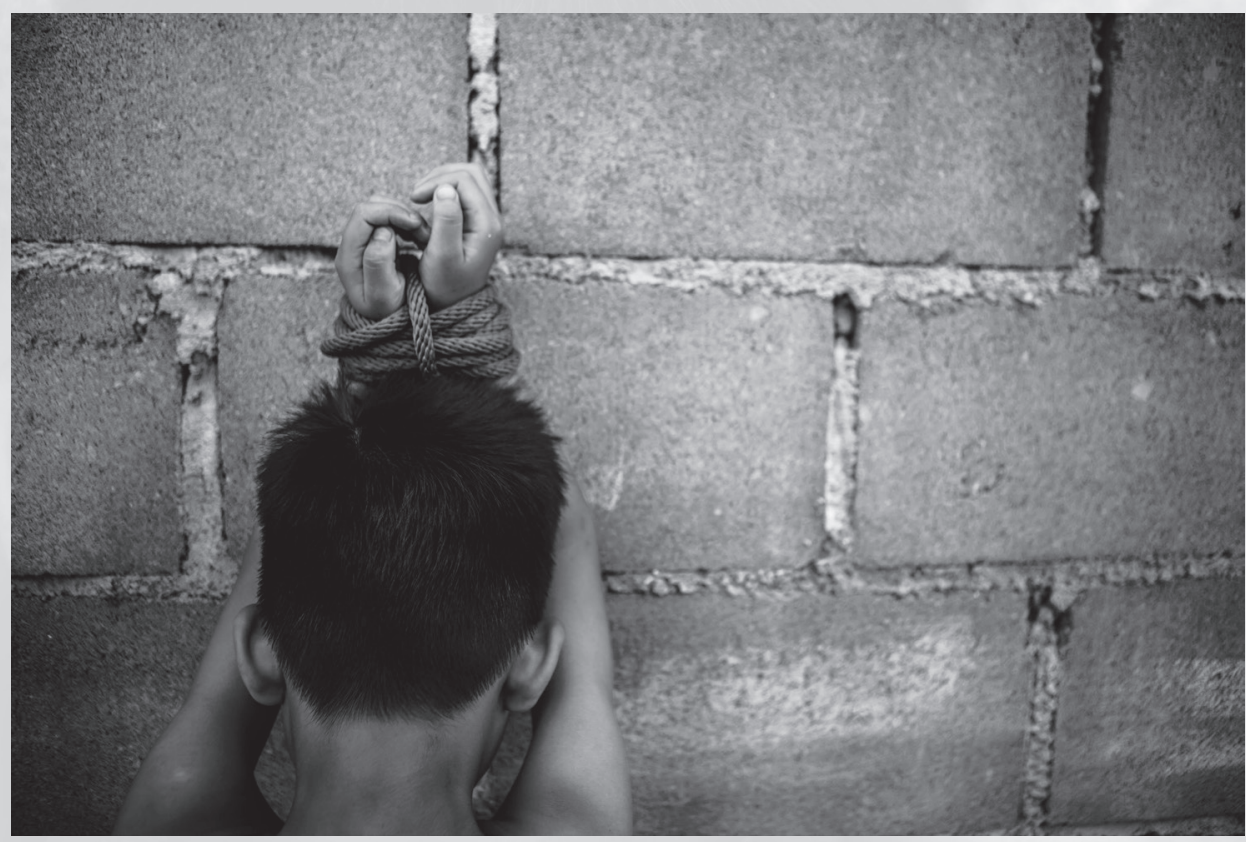





\title{
GENEALOGÍA DE LA TORTURA EN COLOMBIA: UNA MIRADA DESDE LOS DERECHOS HUMANOS*
}

\author{
Paola Alexandra Sierra-Zamora \\ Lina VANessa JimÉnEZ-BarRera \\ Universidad Católica de Colombia
}

\section{Resumen}

Con la presente investigación se busca analizar los lineamientos del Estado sobre la tortura de niños, niñas y adolescentes (en adelante, NNA) en Colombia. Se enfoca en el concepto que se tiene sobre tortura para focalizar la problemática de las violaciones de los derechos humanos (en adelante, DDHH); en segunda instancia, se analizan los fallos de la Corte Interamericana de Derechos Humanos (en adelante, Corte IDH) respecto a la tortura; luego se estudiarán la legislación nacional y la jurisprudencia, con el propósito de evidenciar la problemática de NNA, para finalizar unas breves conclusiones.

Palabras clave: lineamientos, Estado, tortura, derechos humanos, tratados, jurisprudencia, normatividad, Colombia.

El autor: Paola Alexandra Sierra-Zamora, docente investigadora de la Facultad de Derecho de la Universidad Católica de Colombia, del grupo de investigación Personas, instituciones y exigencias de justicia. Correo electrónico: pasierra@ucatolica.edu.co

Lina Vanessa Jiménez-Barrera, estudiante de la Facultad de Derecho de la Universidad Católica de Colombia y miembro del semillero de investigación Observatorio de justicia constitucional de derechos humanos, vinculado al Centro de Investigaciones Sociojurídicas (Cisjuc) de la Universidad Católica de Colombia. Correo electrónico: lvjimenez37@ucatolica.edu.co

Recibido: 6 de noviembre de 2018; evaluado: 3 de diciembre de 2018; aceptado: 11 de marzo de 2019

\footnotetext{
Este artículo expone los resultados del proyecto de investigación titulado "Desafíos contemporáneos para la protección de derechos humanos en escenarios de posconflicto, fase II", que forma parte de la línea de investigación Fundamentación e implementación de los derechos humanos, del grupo de investigación Persona, instituciones y exigencias de justicia, reconocido y categorizado como Tipo Al por Colciencias y registrado con el código COL0120899. El grupo está vinculado al Centro de Investigaciones Sociojurídicas (Cisjuc), adscrito a la Facultad de Derecho de la Universidad Católica de Colombia y financiado por el Departamento administrativo de ciencia, tecnología e innovación, Colciencias.
} 


\title{
GENEALOGY OF TORTURE IN COLOMBIA FROM A HUMAN RIGHTS PERSPECTIVE
}

\author{
Paola Alexandra Sierra-Zamora \\ Lina VAnessa Jiménez-Barrera \\ Universidad Católica de Colombia
}

\begin{abstract}
This research seeks to analyze state guidelines on the torture of children and adolescents in Colombia. First, it analyzes the concept of torture in order to focus on problems regarding human rights violations. Second, it examines the judgments of the Inter-American Court of Human Rights regarding torture, followed by a study of national and jurisprudential legislations, aiming to highlight problems regarding children and adolescents. The paper finally presents some brief conclusions.
\end{abstract}

Keywords: guidelines, State, torture, human rights, treaties, jurisprudence, regulations.

About the authors: Paola Alexandra Sierra-Zamora, research professor at the Faculty of Law of the Universidad Católica de Colombia, member of the Research Group "People, institutions, and demands of justice." Email: pasierra@ucatolica.edu.co

Lina Vanessa Jiménez-Barrera, student at the Faculty of Law of the Universidad Católica de Colombia, and member of the Research Hubble "Observatory of Constitutional Justice and Human Rights," linked to the Center for Socio-Legal Research (Cisjuc) at the Universidad Católica de Colombia. Email: lvjimenez37@ucatolica.edu.co

Received: November 6, 2018; reviewed: December 3, 2018; accepted: March 11, 2019 
La dignidad es el respeto que una persona tiene de sí misma y quien la tiene no puede hacer nada que lo vuelva despreciable a sus propios ojos. ${ }^{1}$

\section{Introducción}

En Colombia se busca la estabilidad de los principios de igualdad, justicia, equidad y libertad, con base en los derechos fundamentales del individuo establecidos en la Constitución Política. Se resalta la problemática en la que se encuentran NNA, puesto que son una población vulnerable en el caso de tortura y otras violaciones.

El objetivo propuesto para la presente investigación es reflexionar a partir de un análisis de los lineamientos que ha propuesto el Estado colombiano para proteger a NNA. Se desarrolla en torno a cuatro ejes temáticos: el primero consiste en revisar la generalidad sobre el concepto de tortura de acuerdo con la doctrina y la problemática en la que se encuentran NNA en Colombia. En el segundo se analiza la situación internacional de NNA, la protección que les brinda la Corte IDH, jurisprudencia y los tratados que abordan el problema. En tercera instancia se examina si la Corte Constitucional o la Corte Suprema de Justicia vela por esta problemática actual, ya que esta vulnera los derechos humanos y ocasiona daño físico y psicológico, con lo que se denigra la dignidad humana. Para finalizar, se exponen algunas conclusiones.

Se pretende resolver la siguiente pregunta: ¿Cuáles son los lineamientos que sigue el Estado colombiano frente a la tortura de NNA?

La metodología empleada es de tipo doctrinal hermenéutica, ya que mediante la revisión y el análisis de textos, cifras estadísticas e informes sectoriales se construye un análisis de microproblemas que responde al desarrollo de cada capítulo de la investigación.

\section{Generalidades sobre la tortura}

La tortura es definida en el Código Penal colombiano (art. 178) como una práctica social que consiste en hacer daño a una persona para obtener información mediante la fuerza, que causa daño físico o mental.

\footnotetext{
Concepción Arenal, "La mujer del porvenir", http://www.cervantesvirtual.com/obra-visor/la-mujer-delporvenir--2/html/feec0b8a-82b1-11df-acc7-002185ce6064_2.html\#I_0_ (acceso diciembre 16, 2018).
} 
En la Constitución colombiana de 1991 comienza a fomentarse el respeto a la dignidad humana, como derecho fundamental de cada individuo; se ajustó el Código Penal de acuerdo con las necesidades que se presentaban y se castigaba cada vez con más rigidez el acto de la tortura.

[... la la conducta de tortura no sólo puede predicarse del Estado sino también de los particulares. Por ello, no solo debe sancionarse al Estado, sino también a los particulares, cuando quiera que la cometan. La tortura es una de las muchas formas como se puede vulnerar el derecho a la integridad personal. Los tratos y las penas crueles, inhumanas o degradantes son, por ejemplo, otras formas de vulnerar ese derecho. ${ }^{2}$

La práctica de la tortura es inaceptable en cualquier circunstancia, incluso si el Estado es quien la usa. Esta condena es tan fuerte que ha llegado a ser aceptada internacionalmente y se convierte en un principio fundamental del derecho.

El tratado internacional para prevenir y sancionar la tortura define:

Para los efectos de la presente Convención se entenderá por tortura todo acto realizado intencionalmente por el cual se inflija a una persona penas o sufrimientos físicos o mentales, con fines de investigación criminal, como medio intimidatorio, como castigo personal, como medida preventiva, como pena o con cualquier otro fin. Se entenderá también como tortura la aplicación sobre una persona de métodos tendientes a anular la personalidad de la víctima o a disminuir su capacidad física o mental, aunque no causen dolor físico o angustia psíquica. ${ }^{3}$

Esta práctica social es frecuente en la actualidad. A raíz de esta problemática, las principales normas internacionales y colombianas están orientadas a la implementación y aplicación efectiva en el orden jurídico de los más altos estándares internacionales contra la tortura y tratos o penas crueles e inhumanas.

Los métodos de tortura han variado y se han profesionalizado con el avance de la ciencia y de la tecnología; ya no se habla solo de lesiones físicas ostensibles, sino también de lesiones psicológicas que pueden ser permanentes.

Corte Constitucional, Sentencia C-587 de 12 de noviembre de 1992, M. P. Ciro Angarita Barón.

Comisión interamericana de derechos humanos, Convención interamericana para prevenir y sancionar la tortura (Cartagena de Indias, 9 de diciembre de 1985), art. 2. 


\subsection{Tortura en niños, niñas y adolescentes (NNA)}

En Colombia, NNA son ciudadanos plenos sujetos de derecho, acogidos a un entorno social y cultural, entendidos como sujetos que están en proceso de desarrollo. Esto significará que son capaces, que pueden participar en lo que atañe a su vida y según su momento de desarrollo y que deben ser valorados en el presente y no solo como un desarrollo a futuro.

No obstante, las problemáticas del conflicto armado en el que se encuentra el Estado colombiano afectan a NNA, con crímenes de diferente índole que vulneran los derechos humanos. Son delitos de lesa humanidad, con los que se viola el concepto que se tiene de justicia en la población colombiana:

La justicia es entendida desde un punto social y jurídico que surge de la introducción de la dignidad humana como factor esencial intrínseco y del respeto al otro por el hecho de ser mi igual, sin hacer juicios de valor que lo califiquen como seudo humano. De esta forma, se pretende expresar la intolerabilidad de categorizaciones en cuanto al sujeto. ${ }^{4}$

Conforman el grupo más vulnerable en cuestión de defensa de los derechos humanos, puesto que muchas de las víctimas de tortura se abstienen de denunciar los hechos debido a las múltiples dificultades para acceder a la justicia, al temor por la presencia y el control de los victimarios en amplias zonas del país y a la ausencia de mecanismos de protección a favor de las víctimas. ${ }^{5}$

Asimismo, la tortura de NNA ocurre en distintos contextos, por ejemplo, en allanamientos por parte de la Policía, porque son percibidos como causantes de amenazas contra el orden público o la seguridad; cuando están encarcelados o en centros de detención; y cuando son percibidos como vinculados de cualquier forma a grupos subversivos, incluso por ser hijos de militantes.

$4 \quad$ Astrid Rocío Galán Galindo, "Los derechos humanos fundamentados mediante la legitimación y la moral jurídica", Novum Jus 10, núm. 1 (enero 2016): 33, https://editorial.ucatolica.edu.co/ojsucatolica/revistas_ucatolica/index.php/Juridica/article/view/1175/1162 (acceso diciembre 16, 2018).

5 Es un ente interinstitucional que busca potenciar el trabajo de organizaciones defensoras de derechos humanos, y promueve acciones dirigidas a prevención, protección, recuperación de las víctimas de tortura y difusión de la situación de la problemática en instancias nacionales e internacionales. Está conformada por la Asociación de familiares de detenidos y desaparecidos (Asfaddes), Colectivo de abogados José Alvear Restrepo (Cajar) y Comisión colombiana de juristas (CCJ).

Coalición colombiana contra la tortura, Informe $4^{\circ}$ periódico del Estado colombiano al Comité contra la tortura (Bogotá: La Coalición, 2009). 
Asimismo, la tortura de NNA ocurre en distintos contextos, como en allanamientos por parte de la Policía, porque son percibidos como causantes de amenazas contra el orden público o la seguridad, al pertenecer, por ejemplo, a grupos al margen de la ley. En este escenario, es probable que NNA estén expuestos a torturas, palizas, asilamientos, ataduras, violaciones, humillaciones y acoso, situaciones propias del momento.

De acuerdo con la Constitución Política de 1991, la función del Estado es defender aquellos derechos que se vean vulnerados y en representación de este se encuentra el Instituto colombiano de bienestar familiar (ICBF), ${ }^{6}$ el cual no se ha pronunciado frente a esta problemática. En otras palabras, el Estado colombiano está obligado a defender y a proteger los derechos inalienables de NNA, ya que son fundamentales para el desarrollo de la sociedad.

\section{Problemática internacional de NNA}

Desde el punto de vista del ámbito internacional, la Corte IDH salvaguarda los derechos de NNA frente a las acciones de tortura que se presentan en la actualidad. ${ }^{7}$

La Corte IDH alude "a los derechos que corresponden a todos los niños de recibir protección, cuidados y ayuda especiales, bajo el rubro de derecho de protección a la maternidad y a la infancia". ${ }^{8}$ Este reconocimiento que les brinda la Corte se acompaña del establecimiento de un deber por parte de los Estados de ofrecer protección especial y reforzada hacia ellos; de ello se deriva el principio del interés superior de NNA, que supone la obligación de los Estados de adoptar y reforzar sus medidas de protección. Este reconocimiento se ve reflejado en lo estipulado por el Senado y la Cámara de Diputados de Argentina:

6 El Instituto colombiano del bienestar familiar (ICBF) es la entidad del Estado colombiano que trabaja por la prevención y protección integral de la primera infancia, la niñez, la adolescencia y el bienestar de las familias en Colombia. Brinda atención especial a aquellos en condiciones de amenaza e inobservancia o vulneración de sus derechos.

7 Liliana Galdámez, "La noción de tortura en la jurisprudencia de la Corte interamericana de derechos humanos" Cejil, núm. 2 (septiembre 2006): 7, http://www.corteidh.or.cr/tablas/r24796.pdf (acceso diciembre 16, 2018)

8 Gonzalo Aguilar Cavallo, "El principio del interés superior del niño y la Corte interamericana de derechos humanos" Estudios Constitucionales 6, núm. 1 (2008): 89, https://www.redalyc.org/articulo.oa?id=82060110 (acceso diciembre 16, 2018). 
Artículo $9^{\circ}$. Derecho a la dignidad y a la integridad personal.

Las niñas, niños y adolescentes tienen derecho a la dignidad como sujetos de derechos y de personas en desarrollo; a no ser sometidos a trato violento, discriminatorio, vejatorio, humillante, intimidatorio; a no ser sometidos a ninguna forma de explotación económica, torturas, abusos o negligencias, explotación sexual, secuestros o tráfico para cualquier fin o en cualquier forma o condición cruel o degradante. ${ }^{9}$

El Estado colombiano tiene como obligación garantizar a NNA una vida digna para su crecimiento y desarrollo, además de velar y defender aquellos derechos que sean vulnerados, al brindar ayuda frente a los actos o daños que se cometieran.

En efecto, es preciso conocer las etapas de crecimiento, que se encuentran en un rango de 1 a 17 años de edad. Estas permiten reconocer las necesidades básicas y las medidas de protección acordes a su edad, con el fin de mejorar su adaptación con respeto por su autonomía y el ejercicio de sus derechos.

\subsection{Principios de tortura en la jurisprudencia de la Corte IDH}

Si bien todos los instrumentos internacionales contienen similares elementos para la construcción de la noción de tortura, se observan matices o diferencias entre ellos, cuestión que ha sido invocada en ciertos ámbitos para intentar re-definir la tortura y "suavizar" el alcance de la prohibición en la investigación del crimen organizado y el terrorismo. ${ }^{10}$

Para encontrar una posible solución ante esta práctica, la Corte IDH impone penas para castigar a los delincuentes, pero la repetición de los casos sigue en aumento.

La Corte IDH ha indicado que los actos ilícitos como la tortura en NNA son inhumanos y vulneran sus derechos, por lo cual interpone medidas para la no repetición de estos actos, con el propósito de que los Estados protejan los derechos de NNA. Además, la Corte solicita a los Estados mayor exigencia y firmeza para

Congreso de la República Argentina, Ley 26.061, "Ley de protección integral de los derechos de las niñas, niños y adolescentes" (Buenos Aires: Boletín Oficial núm. 30.767, 28 de septiembre de 2005), art. 9.

10 Zulita Fellini, "Estándares internacionales de derechos humanos para la protección de los niños víctimas y testigos en sede judicial" en Acceso a la justicia de niños/as víctimas, ed. Marina Fucito (Buenos Aires: Jufejus, 2016), 31 . 
censurar las violaciones, ya que la integridad personal y la dignidad humanas son valores fundamentales en las sociedades democráticas.

Por otro lado, entre la doctrina emanada de la Corte IDH se propone el uso del derecho internacional, ya que el sistema internacional de derechos humanos consagra la prohibición de la práctica de la tortura, mediante normas especiales para sancionar dichos actos. ${ }^{11}$

\section{Situación NNA en Colombia}

El Estado colombiano debe proteger a todos los ciudadanos, en especial a aquellos que por diferentes circunstancias están en situación de desamparo y debe sancionar a quienes atenten contra los bienes jurídicamente tutelados; sin duda alguna, el acto ilícito de la tortura es uno de los crímenes que mayor rechazo provocan tanto en el ámbito nacional como internacional. En este sentido es relevante, tanto para los efectos de las víctimas como de los procesos que a partir de estos hechos puedan generarse, que los actos de tortura sean calificados como tales y no queden en un terreno incierto como es la afectación genérica de la integridad personal.

"El Estado colombiano no ha buscado asegurar el respeto y garantía de los derechos humanos ni poner fin a las graves y sistemáticas violaciones a los más afectados por el conflicto que se presente en Colombia". ${ }^{12}$ En otras palabras, el Estado colombiano apoya y salvaguarda a muchas personas que lo necesitan, pero en circunstancias un poco más graves, esta protección no se ve con claridad, por ejemplo, no se ha pronunciado sobre la tortura de NNA.

\subsection{Leyes y decretos en Colombia frente a la tortura}

La ley de la tortura no está pronunciada en Colombia, pero la Ley 1453 de 2011 habla sobre las medidas penales para garantizar la seguridad ciudadana, en su Artículo 6, llamado Tráfico de menores; acompañada por la Ley 599 de 2000. Ambas protegen a NNA ante diferentes abusos.

11 Jaime Cubides Cárdenas, Johan Sebastián Suárez Pinilla y Paola Alexandra Sierra Zamora, "El derecho internacional humanitario y la Corte Interamericana de Derechos Humanos en la protección de los derechos de verdad y justicia a las víctimas del conflicto armado interno colombiano" en Cuestiones dialécticas en torno a los derechos humanos y la paz, ed. Jaime Cubides Cárdenas y Antonio Fajardo Rico (Curitiba: Instituto Memória. Centro de Estudos da Contemporaneidade, 2018), 164.

12 Comisión Colombiana de Juristas, Colombia: tortura y otros tratos crueles, inhumanos y degradantes (Bogotá: Comisión Colombiana de Juristas, 2004), 23. 
En la legislación colombiana, el Estado, por medio de Unicef, ${ }^{13}$ ayuda a proteger los derechos de NNA que son víctimas de esta problemática; según el Instituto colombiano de bienestar familiar, la cifra de NNA víctimas de tortura es de 126 millones, sin contar las zonas veredales, en donde los menores de edad son los principales afectados dentro del marco de la violencia.

La terminación del conflicto armado en Colombia permite esclarecer las violaciones de los derechos humanos:

[La] implementación [de los derechos humanos] debe transcurrir en medio de una confrontación armada, con violaciones sistemáticas de derechos humanos frente a actores armados que no han sido derrotados militarmente por las Fuerzas de Seguridad del Estado. Y es que hay que entender, y confrontar, que con la Firma del Acuerdo Final para la terminación del conflicto armado no termina la guerra. Se necesitan tiempo para lograr una adecuada implementación, tiempo para lograr una adecuada sistematización y tiempo para lograr una adecuada armonización, eso sin contar con las adversidades que puedan ocurrir en el tiempo y espacio que requiera. ${ }^{14}$

Uno de los enfoques de esta investigación fue identificar los lineamientos que el Estado colombiano sigue frente a la tortura de NNA; sí existen normas que los protegen, pero falta ser más rígido y estricto a la hora de proteger y castigar esta clase de violaciones en las cuales están viviendo NNA.

\section{Conclusiones}

El Estado no se ha pronunciado en el año 2018 ante las torturas que se han presentado, aunque millones de NNA fueron afectados por el conflicto armado mediante las torturas y otra clase de malos tratos.

13 Unicef es una entidad especializada en la supervivencia y la protección del desarrollo de NNA. Funciona con el objetivo de brindar apoyo técnico y financiero a programas nacionales y comunitarios de educación e intervención que se especializan en la atención de la salud y la nutrición. Entre los aspectos a los que dan prioridad se encuentra la prevención de la tortura y los tratos crueles en la primera infancia.

14 Jaime Cubides Cárdenas, Paola Alexandra Sierra Zamora y María Dolores Núñez Ávila, "El fuero militar en el derecho colombiano vs. el derecho ecuatoriano" en El fuero militar: justicia interamericana y operaciones para el mantenimiento de la paz, ed. Jaime Cubides Cárdenas (Bogotá: Escuela Militar de Cadetes General José María Córdova, 2017), 61. 
El Estado colombiano está obligado por la Constitución Política y los tratados internacionales a defender y proteger a aquellos que están en situaciones de violencia o tortura, pero tras el análisis sobre la jurisprudencia se observa vacío; ello demuestra que por parte del Estado colombiano y las entidades que lo apoyan no existe una investigación a profundidad sobre la tortura de NNA. Además, luego de la firma del acuerdo de paz para la terminación del conflicto, no se ha elaborado un estudio en el que se observe el porcentaje de NNA que sufrieron de tortura y los que hoy la padecen ni sobre las problemáticas ligadas a esta.

Para finalizar, se encuentra que la protección del derecho a la integridad y al desarrollo de NNA ha sido una utopía en el Estado colombiano, ya que la legitimidad de la sanción sobre este acto ilegal no se ha interpuesto de manera concreta.

Los tratados internacionales que propenden por la garantía de los derechos de NNA han sido acogidos por el Estado colombiano; algunos de ellos se encuentran en ejecución y otros no han entrado en vigor. ${ }^{15}$

\section{Referencias}

Aguilar Cavallo, Gonzalo. "El principio del interés superior del niño y la Corte interamericana de derechos humanos". Estudios Constitucionales, núm. 1 (2008): 223-247. https:// www.redalyc.org/articulo.oa?id=82060110 (acceso diciembre 16, 2018).

Arenal, Concepción. "La mujer del porvenir". http://www.cervantesvirtual.com/obra-visor/ la-mujer-del-porvenir--2/html/feec0b8a-82bl-1ldf-acc7-002185ce6064_2.html\#I_0_ (acceso diciembre 16, 2018).

Coalición colombiana contra la tortura. Informe $4^{\circ}$ periódico del Estado colombiano al Comité contra la tortura. Bogotá: La Coalición, 2009.

Comisión Colombiana de Juristas. Colombia: tortura y otros tratos crueles, inhumanos y degradantes. Bogotá: Comisión Colombiana de Juristas, 2004.

Comisión interamericana de derechos humanos. Convención interamericana para prevenir y sancionar la tortura. Cartagena de Indias, 9 de diciembre de 1985.

Congreso de la República Argentina. Ley 26.061, "Ley de protección integral de los derechos de las niñas, niños y adolescentes". Buenos Aires: Boletín Oficial núm. 30.767, 28 de septiembre de 2005.

15 Jaime Cubides Cárdenas, Tania Giovanna Vivas Barrera y Paola Andrea Sierra Zamora, "Exordio conclusivo: de la responsabilidad internacional y la protección ambiental" en Responsabilidad internacional y protección ambiental: en tiempos de paz, en medio del conflicto armado y en etapas de posconflicto, ed. Tania Giovanna Vivas Barrera (Bogotá: Universidad Católica de Colombia, 2018), 311-317. 
Congreso de la República de Colombia. Ley 1453 de 2011, "Por medio de la cual se reforma el Código Penal, el Código de Procedimiento Penal, el Código de Infancia y Adolescencia, las reglas sobre extinción de dominio y se dictan otras disposiciones en materia de seguridad”. Bogotá: Diario Oficial núm. 48.110, 24 de junio de 2011.

Congreso de la República de Colombia. Ley 599 de 2000, "Por medio de la cual se crea el Código Penal colombiano”. Bogotá: Diario Oficial núm. 44.097, 24 de julio de 2000. Corte Constitucional. Sentencia C-587 del 12 de noviembre de 1992. M. P. Ciro Angarita Barón. Cubides Cárdenas Jaime, Paola Alexandra Sierra Zamora y Hugo Carrasco Soulé. "El control de convencionalidad: aspectos generales en el sistema interamericano de derechos humanos y en el derecho colombiano" en El control de convencionalidad (CCV): fundamentación e implementación desde el sistema interamericano de derechos humanos, editado por Jaime Cubides Cárdenas, 41-75. Bogotá: Universidad Católica de Colombia, 2016.

Cubides Cárdenas Jaime, Paola Alexandra Sierra Zamora y María Dolores Núñez Ávila. "El fuero militar en el derecho colombiano vs. el derecho ecuatoriano" en El fuero militar: justicia interamericana y operaciones para el mantenimiento de la paz, editado por Jaime Cubides Cárdenas, 65-81. Bogotá: Escuela Militar de Cadetes General José María Córdova, 2017.

Cubides Cárdenas, Jaime, Johan Sebastián Suárez Pinilla y Paola Alexandra Sierra Zamora, "El derecho internacional humanitario y la corte interamericana de derechos humanos en la protección de los derechos de verdad u justicia a las víctimas del conflicto armado interno colombiano" en Cuestiones dialécticas en torno a los derechos humanos y la paz, editado por Jaime Cubides Cárdenas y Antonio Fajardo Rico, 164-186. Curitiba: Instituto Memória. Centro de Estudos da Contemporaneidade, 2018.

Cubides Cárdenas, Jaime, Tania Giovanna Vivas Barrera y Paola Andrea Sierra Zamora. "Exordio conclusivo: de la responsabilidad internacional y la protección ambiental" en Responsabilidad internacional y protección ambiental: en tiempos de paz, en medio del conflicto armado y en etapas de posconflicto, editado por Tania Giovanna Vivas Barrera, 311-316. Bogotá: Universidad Católica de Colombia, 2018.

Fellini, Zulita. "Estándares internacionales de derechos humanos para la protección de los niños víctimas y testigos en sede judicial" en Acceso a la justicia de niños/as víctimas, editado por Marina Fucito, 31-46. Buenos Aires: Jufejus, 2016.

Galán Galindo, Astrid Rocío. "Los derechos humanos fundamentados mediante la legitimación y la moral jurídica”. Novum Jus 10, núm. 1 (enero 2016): 33. https://editorial.ucatolica. edu.co/ojsucatolica/revistas_ucatolica/index.php/Juridica/article/view/1175/1162 (acceso diciembre 16, 2018). 
Galdámez, Liliana. "La noción de tortura en la jurisprudencia de la Corte interamericana de derechos humanos". Cejil, núm. 2 (septiembre 2006): 89-100. http://www.corteidh. or.cr/tablas/r24796.pdf (acceso diciembre 16, 2018).

República de Colombia. Constitución Política. Bogotá: Legis, 1991. 